\title{
Existence and multiplicity of positive solutions for a system of fractional boundary value problems
}

\author{
Johnny Henderson ${ }^{1}$ and Rodica Luca2 ${ }^{2 *}$
}

${ }^{\text {"Correspondence: }}$
rluca@math.tuiasi.ro
${ }^{2}$ Department of Mathematics,
Gh. Asachi Technical University, lasi,
700506, Romania
Full list of author information is
available at the end of the article

available at the end of the article

\begin{abstract}
We study the existence and multiplicity of positive solutions for a system of nonlinear Riemann-Liouville fractional differential equations, subject to integral boundary conditions. The nonsingular and singular cases for the nonlinearities are investigated.
\end{abstract} MSC: $34 \mathrm{A0} 08 ; 45 \mathrm{G} 15$

Keywords: Riemann-Liouville fractional differential equation; integral boundary conditions; positive solutions

\section{Introduction}

We consider the system of nonlinear ordinary fractional differential equations

$$
\begin{cases}D_{0+}^{\alpha} u(t)+f(t, v(t))=0, & t \in(0,1), n-1<\alpha \leq n, \\ D_{0+}^{\beta} v(t)+g(t, u(t))=0, & t \in(0,1), m-1<\beta \leq m,\end{cases}
$$

with the integral boundary conditions

$$
\text { (BC) } \begin{cases}u(0)=u^{\prime}(0)=\cdots=u^{(n-2)}(0)=0, & u(1)=\int_{0}^{1} u(s) d H(s), \\ v(0)=v^{\prime}(0)=\cdots=v^{(m-2)}(0)=0, & v(1)=\int_{0}^{1} v(s) d K(s),\end{cases}
$$

where $n, m \in \mathbb{N}, n, m \geq 2, D_{0+}^{\alpha}$ and $D_{0_{+}}^{\beta}$ denote the Riemann-Liouville derivatives of orders $\alpha$ and $\beta$, respectively, and the integrals from (BC) are Riemann-Stieltjes integrals.

Under sufficient conditions on functions $f$ and $g$, which can be nonsingular or singular in the points $t=0$ and/or $t=1$, we study the existence and multiplicity of positive solutions of problem (S)-(BC). We use the Guo-Krasnosel'skii fixed point theorem (see [1]) and some theorems from the fixed point index theory (from [2] and [3]). By a positive solution of problem $(\mathrm{S})-(\mathrm{BC})$ we mean a pair of functions $(u, v) \in C([0,1]) \times C([0,1])$ satisfying $(\mathrm{S})$ and (BC) with $u(t) \geq 0, v(t) \geq 0$ for all $t \in[0,1]$ and $\sup _{t \in[0,1]} u(t)>0, \sup _{t \in[0,1]} v(t)>0$. The system (S) with $\alpha=n, \beta=m$ and the boundary conditions (BC) where $H$ and $K$ are scale functions (that is, multi-point boundary conditions) has been investigated in [4] (the nonsingular case) and [5] (the singular case). In [6], the authors give sufficient conditions for $\lambda, \mu, f$, and $g$ such that the system

$$
\left(\mathrm{S}_{1}\right) \quad \begin{cases}D_{0+}^{\alpha} u(t)+\lambda f(t, u(t), v(t))=0, & t \in(0,1), n-1<\alpha \leq n, \\ D_{0+}^{\beta} v(t)+\mu g(t, u(t), v(t))=0, & t \in(0,1), m-1<\beta \leq m,\end{cases}
$$

\section{Springer}

O2014 Henderson and Luca; licensee Springer. This is an Open Access article distributed under the terms of the Creative Commons Attribution License (http://creativecommons.org/licenses/by/2.0), which permits unrestricted use, distribution, and reproduction in any medium, provided the original work is properly cited. 
with the boundary conditions (BC) with $H$ and $K$ scale functions, has positive solutions $(u(t) \geq 0, v(t) \geq 0$ for all $t \in[0,1]$, and $(u, v) \neq(0,0))$.

Fractional differential equations describe many phenomena in various fields of engineering and scientific disciplines such as physics, biophysics, chemistry, biology, economics, control theory, signal and image processing, aerodynamics, viscoelasticity, electromagnetics, and so on (see [7-13]).

In Section 2, we present the necessary definitions and properties from the fractional calculus theory and some auxiliary results dealing with a nonlocal boundary value problem for fractional differential equations. In Section 3, we give some existence and multiplicity results for positive solutions with respect to a cone for our problem (S)-(BC), where $f$ and $g$ are nonsingular functions. The case when $f$ and $g$ are singular at $t=0$ and/or $t=1$ is studied in Section 4. Finally, in Section 5, we present two examples which illustrate our main results.

\section{Preliminaries and auxiliary results}

We present here the definitions, some lemmas from the theory of fractional calculus and some auxiliary results that will be used to prove our main theorems.

Definition 2.1 The (left-sided) fractional integral of order $\alpha>0$ of a function $f:(0, \infty) \rightarrow$ $\mathbb{R}$ is given by

$$
\left(I_{0+}^{\alpha} f\right)(t)=\frac{1}{\Gamma(\alpha)} \int_{0}^{t}(t-s)^{\alpha-1} f(s) d s, \quad t>0,
$$

provided the right-hand side is pointwise defined on $(0, \infty)$, where $\Gamma(\alpha)$ is the Euler gamma function defined by $\Gamma(\alpha)=\int_{0}^{\infty} t^{\alpha-1} e^{-t} d t, \alpha>0$.

Definition 2.2 The Riemann-Liouville fractional derivative of order $\alpha \geq 0$ for a function $f:(0, \infty) \rightarrow \mathbb{R}$ is given by

$$
\left(D_{0+}^{\alpha} f\right)(t)=\left(\frac{d}{d t}\right)^{n}\left(I_{0+}^{n-\alpha} f\right)(t)=\frac{1}{\Gamma(n-\alpha)}\left(\frac{d}{d t}\right)^{n} \int_{0}^{t} \frac{f(s)}{(t-s)^{\alpha-n+1}} d s, \quad t>0
$$

where $n=\llbracket \alpha \rrbracket+1$, provided that the right-hand side is pointwise defined on $(0, \infty)$.

The notation $\llbracket \alpha \rrbracket$ stands for the largest integer not greater than $\alpha$. We also denote the Riemann-Liouville fractional derivative of $f$ by $D_{0+}^{\alpha} f(t)$. If $\alpha=m \in \mathbb{N}$ then $D_{0+}^{m} f(t)=f^{(m)}(t)$ for $t>0$, and if $\alpha=0$ then $D_{0+}^{0} f(t)=f(t)$ for $t>0$.

Lemma 2.1 ([10]) Let $\alpha>0$ and $n=\llbracket \alpha \rrbracket+1$ for $\alpha \notin \mathbb{N}$ and $n=\alpha$ for $\alpha \in \mathbb{N}$; that is, $n$ is the smallest integer greater than or equal to $\alpha$. Then the solutions of the fractional differential equation $D_{0+}^{\alpha} u(t)=0,0<t<1$, are

$$
u(t)=c_{1} t^{\alpha-1}+c_{2} t^{\alpha-2}+\cdots+c_{n} t^{\alpha-n}, \quad 0<t<1,
$$

where $c_{1}, c_{2}, \ldots, c_{n}$ are arbitrary real constants. 
Lemma 2.2 $([6,10])$ Let $\alpha>0, n$ be the smallest integer greater than or equal to $\alpha(n-1<$ $\alpha \leq n)$ and $y \in L^{1}(0,1)$. The solutions of the fractional equation $D_{0+}^{\alpha} u(t)+y(t)=0,0<t<1$, are

$$
u(t)=-\frac{1}{\Gamma(\alpha)} \int_{0}^{t}(t-s)^{\alpha-1} y(s) d s+c_{1} t^{\alpha-1}+\cdots+c_{n} t^{\alpha-n}, \quad 0<t<1,
$$

where $c_{1}, c_{2}, \ldots, c_{n}$ are arbitrary real constants.

We consider now the fractional differential equation

$$
D_{0+}^{\alpha} u(t)+y(t)=0, \quad 0<t<1, n-1<\alpha \leq n,
$$

with the integral boundary conditions

$$
u(0)=u^{\prime}(0)=\cdots=u^{(n-2)}(0)=0, \quad u(1)=\int_{0}^{1} u(s) d H(s)
$$

where $n \in \mathbb{N}, n \geq 2$, and $H:[0,1] \rightarrow \mathbb{R}$ is a function of the bounded variation.

Lemma 2.3 If $H:[0,1] \rightarrow \mathbb{R}$ is a function of bounded variation, $\Delta_{1}=1-\int_{0}^{1} s^{\alpha-1} d H(s) \neq 0$ and $y \in C([0,1])$, then the solution of problem (1)-(2) is given by

$$
\begin{aligned}
u(t)= & -\frac{1}{\Gamma(\alpha)} \int_{0}^{t}(t-s)^{\alpha-1} y(s) d s+\frac{t^{\alpha-1}}{\Delta_{1} \Gamma(\alpha)}\left[\int_{0}^{1}(1-s)^{\alpha-1} y(s) d s\right. \\
& \left.-\int_{0}^{1}\left(\int_{s}^{1}(\tau-s)^{\alpha-1} d H(\tau)\right) y(s) d s\right], \quad 0 \leq t \leq 1 .
\end{aligned}
$$

Proof By Lemma 2.2, the solutions of equation (1) are

$$
u(t)=-\frac{1}{\Gamma(\alpha)} \int_{0}^{t}(t-s)^{\alpha-1} y(s) d s+c_{1} t^{\alpha-1}+\cdots+c_{n} t^{\alpha-n},
$$

where $c_{1}, \ldots, c_{n} \in \mathbb{R}$. By using the conditions $u(0)=u^{\prime}(0)=\cdots=u^{(n-2)}(0)=0$, we obtain $c_{2}=\cdots=c_{n}=0$. Then we conclude

$$
u(t)=c_{1} t^{\alpha-1}-\frac{1}{\Gamma(\alpha)} \int_{0}^{t}(t-s)^{\alpha-1} y(s) d s .
$$

Now, by condition $u(1)=\int_{0}^{1} u(s) d H(s)$, we deduce

$$
c_{1}-\frac{1}{\Gamma(\alpha)} \int_{0}^{1}(1-s)^{\alpha-1} y(s) d s=\int_{0}^{1}\left[c_{1} s^{\alpha-1}-\frac{1}{\Gamma(\alpha)} \int_{0}^{s}(s-\tau)^{\alpha-1} y(\tau) d \tau\right] d H(s)
$$

or

$$
\begin{aligned}
c_{1}\left(1-\int_{0}^{1} s^{\alpha-1} d H(s)\right)= & \frac{1}{\Gamma(\alpha)} \int_{0}^{1}(1-s)^{\alpha-1} y(s) d s \\
& -\frac{1}{\Gamma(\alpha)} \int_{0}^{1}\left(\int_{0}^{s}(s-\tau)^{\alpha-1} y(\tau) d \tau\right) d H(s) .
\end{aligned}
$$


Henderson and Luca Boundary Value Problems 2014, 2014:60

Page 4 of 17

http://www.boundaryvalueproblems.com/content/2014/1/60

So, we obtain

$$
\begin{aligned}
c_{1} & =\frac{1}{\Delta_{1} \Gamma(\alpha)} \int_{0}^{1}(1-s)^{\alpha-1} y(s) d s-\frac{1}{\Delta_{1} \Gamma(\alpha)} \int_{0}^{1}\left(\int_{0}^{s}(s-\tau)^{\alpha-1} y(\tau) d \tau\right) d H(s) \\
& =\frac{1}{\Delta_{1} \Gamma(\alpha)} \int_{0}^{1}(1-s)^{\alpha-1} y(s) d s-\frac{1}{\Delta_{1} \Gamma(\alpha)} \int_{0}^{1}\left(\int_{\tau}^{1}(s-\tau)^{\alpha-1} d H(s)\right) y(\tau) d \tau \\
& =\frac{1}{\Delta_{1} \Gamma(\alpha)} \int_{0}^{1}(1-s)^{\alpha-1} y(s) d s-\frac{1}{\Delta_{1} \Gamma(\alpha)} \int_{0}^{1}\left(\int_{s}^{1}(\tau-s)^{\alpha-1} d H(\tau)\right) y(s) d s .
\end{aligned}
$$

Therefore, we get the expression (3) for the solution of problem (1)-(2).

Lemma 2.4 Under the assumptions of Lemma 2.3, the Green's function for the boundary value problem (1)-(2) is given by

$$
G_{1}(t, s)=g_{1}(t, s)+\frac{t^{\alpha-1}}{\Delta_{1}} \int_{0}^{1} g_{1}(\tau, s) d H(\tau), \quad(t, s) \in[0,1] \times[0,1],
$$

where

$$
g_{1}(t, s)=\frac{1}{\Gamma(\alpha)} \begin{cases}t^{\alpha-1}(1-s)^{\alpha-1}-(t-s)^{\alpha-1}, & 0 \leq s \leq t \leq 1 \\ t^{\alpha-1}(1-s)^{\alpha-1}, & 0 \leq t \leq s \leq 1\end{cases}
$$

Proof By Lemma 2.3 and relation (3), we conclude

$$
\begin{aligned}
u(t)= & \frac{1}{\Gamma(\alpha)}\left\{\int_{0}^{t}\left[t^{\alpha-1}(1-s)^{\alpha-1}-(t-s)^{\alpha-1}\right] y(s) d s+\int_{t}^{1} t^{\alpha-1}(1-s)^{\alpha-1} y(s) d s\right. \\
& -\int_{0}^{1} t^{\alpha-1}(1-s)^{\alpha-1} y(s) d s+\frac{t^{\alpha-1}}{\Delta_{1}}\left[\int_{0}^{1}(1-s)^{\alpha-1} y(s) d s\right. \\
& \left.\left.-\int_{0}^{1}\left(\int_{s}^{1}(\tau-s)^{\alpha-1} d H(\tau)\right) y(s) d s\right]\right\} \\
= & \frac{1}{\Gamma(\alpha)}\left\{\int_{0}^{t}\left[t^{\alpha-1}(1-s)^{\alpha-1}-(t-s)^{\alpha-1}\right] y(s) d s\right. \\
& +\int_{t}^{1} t^{\alpha-1}(1-s)^{\alpha-1} y(s) d s-\frac{1}{\Delta_{1}}\left(1-\int_{0}^{1} \tau^{\alpha-1} d H(\tau)\right) \\
& \times \int_{0}^{1} t^{\alpha-1}(1-s)^{\alpha-1} y(s) d s \\
& \left.+\frac{t^{\alpha-1}}{\Delta_{1}}\left[\int_{0}^{1}(1-s)^{\alpha-1} y(s) d s-\int_{0}^{1}\left(\int_{s}^{1}(\tau-s)^{\alpha-1} d H(\tau)\right) y(s) d s\right]\right\} \\
= & \frac{1}{\Gamma(\alpha)}\left\{\int_{0}^{t}\left[t^{\alpha-1}(1-s)^{\alpha-1}-(t-s)^{\alpha-1}\right] y(s) d s+\int_{t}^{1} t^{\alpha-1}(1-s)^{\alpha-1} y(s) d s\right. \\
= & \frac{1}{\Gamma(\alpha)}\left\{\int_{0}^{t}\left[t^{\alpha-1}(1-s)^{\alpha-1}-(t-s)^{\alpha-1}\right] y(s) d s+\int_{t}^{1} t^{\alpha-1}(1-s)^{\alpha-1} y(s) d s\right. \\
& \left.\left.-\int_{0}^{1}\left(\int_{0}^{1}(\tau-s)^{\alpha-1} d H(\tau)\right) y(s) d s\right]\right\} \\
& \left.1 \tau^{\alpha-1}(1-s)^{\alpha-1} d H(\tau)\right) y(s) d s
\end{aligned}
$$




$$
\begin{aligned}
& +\frac{t^{\alpha-1}}{\Delta_{1}}\left[\int_{0}^{1}\left(\int_{0}^{s} \tau^{\alpha-1}(1-s)^{\alpha-1} d H(\tau)\right) y(s) d s\right. \\
& \left.\left.+\int_{0}^{1}\left(\int_{s}^{1}\left[\tau^{\alpha-1}(1-s)^{\alpha-1}-(\tau-s)^{\alpha-1}\right] d H(\tau)\right) y(s) d s\right]\right\} \\
& =\int_{0}^{1} g_{1}(t, s) y(s) d s+\frac{t^{\alpha-1}}{\Delta_{1}} \int_{0}^{1}\left(\int_{0}^{1} g_{1}(\tau, s) d H(\tau)\right) y(s) d s \\
& =\int_{0}^{1} G_{1}(t, s) y(s) d s,
\end{aligned}
$$

where $g_{1}$ and $G_{1}$ are given in (5) and (4), respectively. Hence $u(t)=\int_{0}^{1} G_{1}(t, s) y(s) d s$ for all $t \in[0,1]$.

Lemma 2.5 ([6]) The function $g_{1}$ given by (5) has the properties:

(a) $g_{1}:[0,1] \times[0,1] \rightarrow \mathbb{R}_{+}$is a continuous function and $g_{1}(t, s) \geq 0$ for all $(t, s) \in[0,1] \times[0,1]$.

(b) $g_{1}(t, s) \leq g_{1}\left(\theta_{1}(s), s\right)$, for all $(t, s) \in[0,1] \times[0,1]$.

(c) For any $c \in(0,1 / 2)$,

$$
\min _{t \in[c, 1-c]} g_{1}(t, s) \geq \gamma_{1} g_{1}\left(\theta_{1}(s), s\right) \quad \text { for all } s \in[0,1]
$$

where $\gamma_{1}=c^{\alpha-1}, \theta_{1}(s)=s$ if $1<\alpha \leq 2(n=2)$, and

$$
\begin{aligned}
& \theta_{1}(s)= \begin{cases}\frac{s}{1-(1-s)^{\frac{\alpha-1}{\alpha-2}}}, & s \in(0,1], \\
\frac{\alpha-2}{\alpha-1}, & s=0,\end{cases} \\
& \text { if } n-1<\alpha \leq n, n \geq 3 .
\end{aligned}
$$

Lemma 2.6 If $H:[0,1] \rightarrow \mathbb{R}$ is a nondecreasing function and $\Delta_{1}>0$, then the Green's function $G_{1}$ of the problem (1)-(2) is continuous on $[0,1] \times[0,1]$ and satisfies $G_{1}(t, s) \geq 0$ for all $(t, s) \in[0,1] \times[0,1]$. Moreover, if $y \in C([0,1])$ satisfies $y(t) \geq 0$ for all $t \in[0,1]$, then the unique solution $u$ of problem (1)-(2) satisfies $u(t) \geq 0$ for all $t \in[0,1]$.

Proof By using the assumptions of this lemma, we have $G_{1}(t, s) \geq 0$ for all $(t, s) \in[0,1] \times$ $[0,1]$, and so $u(t) \geq 0$ for all $t \in[0,1]$.

Lemma 2.7 Assume that $H:[0,1] \rightarrow \mathbb{R}$ is a nondecreasing function and $\Delta_{1}>0$. Then the Green's function $G_{1}$ of the problem (1)-(2) satisfies the inequalities:

(a) $G_{1}(t, s) \leq J_{1}(s), \forall(t, s) \in[0,1] \times[0,1]$, where

$$
J_{1}(s)=g_{1}\left(\theta_{1}(s), s\right)+\frac{1}{\Delta_{1}} \int_{0}^{1} g_{1}(\tau, s) d H(\tau), \quad s \in[0,1] .
$$

(b) For every $c \in(0,1 / 2)$, we have

$$
\min _{t \in[c, 1-c]} G_{1}(t, s) \geq \gamma_{1} J_{1}(s) \geq \gamma_{1} G_{1}\left(t^{\prime}, s\right), \quad \forall t^{\prime}, s \in[0,1] .
$$


Proof The first inequality (a) is evident. For part (b), for $c \in(0,1 / 2)$ and $t \in[c, 1-c], t^{\prime}, s \in$ $[0,1]$, we deduce

$$
\begin{aligned}
G_{1}(t, s) & \geq c^{\alpha-1} g_{1}\left(\theta_{1}(s), s\right)+\frac{c^{\alpha-1}}{\Delta_{1}} \int_{0}^{1} g_{1}(\tau, s) d H(\tau) \\
& =c^{\alpha-1}\left(g_{1}\left(\theta_{1}(s), s\right)+\frac{1}{\Delta_{1}} \int_{0}^{1} g_{1}(\tau, s) d H(\tau)\right)=\gamma_{1} J_{1}(s) \geq \gamma_{1} G_{1}\left(t^{\prime}, s\right) .
\end{aligned}
$$

Therefore, we obtain the inequalities (b) of this lemma.

Lemma 2.8 Assume that $H:[0,1] \rightarrow \mathbb{R}$ is a nondecreasing function and $\Delta_{1}>0, c \in$ $(0,1 / 2)$, and $y \in C([0,1]), y(t) \geq 0$ for all $t \in[0,1]$. Then the solution $u(t), t \in[0,1]$ of problem (1)-(2) satisfies the inequality $\min _{t \in[c, 1-c]} u(t) \geq \gamma_{1} \max _{t^{\prime} \in[0,1]} u\left(t^{\prime}\right)$.

Proof For $c \in(0,1 / 2), t \in[c, 1-c], t^{\prime} \in[0,1]$, we have

$$
u(t)=\int_{0}^{1} G_{1}(t, s) y(s) d s \geq \gamma_{1} \int_{0}^{1} J_{1}(s) y(s) d s \geq \gamma_{1} \int_{0}^{1} G_{1}\left(t^{\prime}, s\right) y(s) d s=\gamma_{1} u\left(t^{\prime}\right) .
$$

Then we deduce the conclusion of this lemma.

We can also formulate similar results as Lemmas 2.3-2.8 above for the fractional differential equation

$$
D_{0+}^{\beta} v(t)+h(t)=0, \quad 0<t<1, m-1<\beta \leq m,
$$

with the integral boundary conditions

$$
v(0)=v^{\prime}(0)=\cdots=v^{(m-2)}(0)=0, \quad v(1)=\int_{0}^{1} v(s) d K(s),
$$

where $m \in \mathbb{N}, m \geq 2, K:[0,1] \rightarrow \mathbb{R}$ is a nondecreasing function and $h \in C([0,1])$. We denote by $\Delta_{2}, \gamma_{2}, g_{2}, \theta_{2}, G_{2}$, and $J_{2}$ the corresponding constants and functions for the problem (6)-(7) defined in a similar manner as $\Delta_{1}, \gamma_{1}, g_{1}, \theta_{1}, G_{1}$, and $J_{1}$, respectively.

\section{The nonsingular case}

In this section, we investigate the existence and multiplicity of positive solutions for our problem (S)-(BC) under various assumptions on nonsingular functions $f$ and $g$.

We present the basic assumptions that we shall use in the sequel.

(H1) $H, K:[0,1] \rightarrow \mathbb{R}$ are nondecreasing functions, $\Delta_{1}=1-\int_{0}^{1} s^{\alpha-1} d H(s)>0$, $\Delta_{2}=1-\int_{0}^{1} s^{\beta-1} d K(s)>0$.

(H2) The functions $f, g:[0,1] \times[0, \infty) \rightarrow[0, \infty)$ are continuous and $f(t, 0)=g(t, 0)=0$ for all $t \in[0,1]$.

A pair of functions $(u, v) \in C([0,1]) \times C([0,1])$ is a solution for our problem (S)-(BC) if and only if $(u, v) \in C([0,1]) \times C([0,1])$ is a solution for the nonlinear integral system

$$
\left\{\begin{array}{l}
u(t)=\int_{0}^{1} G_{1}(t, s) f\left(s, \int_{0}^{1} G_{2}(s, \tau) g(\tau, u(\tau)) d \tau\right) d s, \quad t \in[0,1], \\
v(t)=\int_{0}^{1} G_{2}(t, s) g(s, u(s)) d s, \quad t \in[0,1] .
\end{array}\right.
$$


We consider the Banach space $X=C([0,1])$ with supremum norm $\|\cdot\|$ and define the cone $P \subset X$ by $P=\{u \in X, u(t) \geq 0, \forall t \in[0,1]\}$.

We also define the operators $\mathcal{A}: P \rightarrow X$ by

$$
(\mathcal{A u})(t)=\int_{0}^{1} G_{1}(t, s) f\left(s, \int_{0}^{1} G_{2}(s, \tau) g(\tau, u(\tau)) d \tau\right) d s, \quad t \in[0,1],
$$

and $\mathcal{B}: P \rightarrow X, \mathcal{C}: P \rightarrow X$ by

$$
(\mathcal{B} u)(t)=\int_{0}^{1} G_{1}(t, s) u(s) d s, \quad(\mathcal{C} u)(t)=\int_{0}^{1} G_{2}(t, s) u(s) d s, \quad t \in[0,1] .
$$

Under the assumptions (H1) and (H2), using also Lemma 2.6, it is easy to see that $\mathcal{A}$, $\mathcal{B}$, and $\mathcal{C}$ are completely continuous from $P$ to $P$. Thus the existence and multiplicity of positive solutions of the system (S)-(BC) are equivalent to the existence and multiplicity of fixed points of the operator $\mathcal{A}$.

Theorem 3.1 Assume that (H1)-(H2) hold. If the functions $f$ and $g$ also satisfy the conditions:

(H3) There exist positive constants $p \in(0,1]$ and $c \in(0,1 / 2)$ such that

(i) $f_{\infty}^{i}=\liminf _{u \rightarrow \infty} \inf _{t \in[c, 1-c]} \frac{f(t, u)}{u^{p}} \in(0, \infty]$;

(ii) $g_{\infty}^{i}=\liminf _{u \rightarrow \infty} \inf _{t \in[c, 1-c]} \frac{g(t, u)}{u^{1 / p}}=\infty$.

(H4) There exists a positive constant $q \in(0, \infty)$ such that

(i) $f_{0}^{s}=\limsup _{u \rightarrow 0^{+}} \sup _{t \in[0,1]} \frac{f(t, u)}{u^{q}} \in[0, \infty)$;

(ii) $g_{0}^{s}=\limsup _{u \rightarrow 0^{+}} \sup _{t \in[0,1]} \frac{g(t, u)}{u^{1 / q}}=0$,

then the problem (S)-(BC) has at least one positive solution $(u(t), v(t)), t \in[0,1]$.

Proof Because the proof of the theorem is similar to that of Theorem 3.1 from [4], we will sketch some parts of it. From assumption (i) of (H3), we deduce that there exist $C_{1}, C_{2}>0$ such that

$$
f(t, u) \geq C_{1} u^{p}-C_{2}, \quad \forall(t, u) \in[c, 1-c] \times[0, \infty) .
$$

Then for $u \in P$, by using (8), Lemma 2.6, and Lemma 2.7, we obtain after some computations

$$
(\mathcal{A} u)(t) \geq C_{1} \int_{c}^{1-c} G_{1}(t, s)\left(\int_{c}^{1-c}\left(G_{2}(s, \tau)\right)^{p}(g(\tau, u(\tau)))^{p} d \tau\right) d s-C_{3}, \quad \forall t \in[0,1], \quad(9)
$$

where $C_{3}=C_{2} \int_{c}^{1-c} J_{1}(s) d s$. 
For $c$ given in (H3), we define the cone $P_{0}=\left\{u \in P, \inf _{t \in[c, 1-c]} u(t) \geq \gamma\|u\|\right\}$, where $\gamma=$ $\min \left\{\gamma_{1}, \gamma_{2}\right\}$. From our assumptions and Lemma 2.8 , for any $y \in P$, we can easily show that $u=\mathcal{B} y \in P_{0}$ and $v=\mathcal{C} y \in P_{0}$, that is, $\mathcal{B}(P) \subset P_{0}$ and $\mathcal{C}(P) \subset P_{0}$.

We now consider the function $u_{0}(t)=\int_{0}^{1} G_{1}(t, s) d s=\left(\mathcal{B} y_{0}\right)(t) \geq 0, t \in[0,1]$, with $y_{0}(t)=1$ for all $t \in[0,1]$. We define the set

$$
M=\left\{u \in P \text {; there exists } \lambda \geq 0 \text { such that } u=\mathcal{A} u+\lambda u_{0}\right\} .
$$

We will show that $M \subset P_{0}$ and $M$ is a bounded subset of $X$. If $u \in M$, then there exists $\lambda \geq 0$ such that $u(t)=(\mathcal{A} u)(t)+\lambda u_{0}(t), t \in[0,1]$. From the definition of $u_{0}$, we have

$$
u(t)=(\mathcal{A} u)(t)+\lambda\left(\mathcal{B} y_{0}\right)(t)=\mathcal{B}(F u(t))+\lambda\left(\mathcal{B} y_{0}\right)(t)=\mathcal{B}\left(F u(t)+\lambda y_{0}(t)\right) \in P_{0}
$$

where $F: P \rightarrow P$ is defined by $(F u)(t)=f\left(t, \int_{0}^{1} G_{2}(t, s) g(s, u(s)) d s\right)$. Therefore, $M \subset P_{0}$, and from the definition of $P_{0}$, we get

$$
\|u\| \leq \frac{1}{\gamma} \inf _{t \in[c, 1-c]} u(t), \quad \forall u \in M .
$$

From (ii) of assumption (H3), we conclude that for $\varepsilon_{0}=\left(2 /\left(C_{1} m_{1} m_{2} \gamma_{1} \gamma_{2}^{p}\right)\right)^{1 / p}>0$ there exists $C_{4}>0$ such that

$$
(g(t, u))^{p} \geq \varepsilon_{0}^{p} u-C_{4}, \quad \forall(t, u) \in[c, 1-c] \times[0, \infty)
$$

where $m_{1}=\int_{c}^{1-c} J_{1}(\tau) d \tau>0, m_{2}=\int_{c}^{1-c}\left(J_{2}(\tau)\right)^{p} d \tau>0$.

For $u \in M$ and $t \in[c, 1-c]$, by using Lemma 2.7 and the relations (9) and (11), it follows that

$$
\begin{aligned}
u(t) & =(\mathcal{A} u)(t)+\lambda u_{0}(t) \geq(\mathcal{A u} u(t) \\
& \geq C_{1} \gamma_{1} \gamma_{2}^{p} \int_{c}^{1-c} J_{1}(s)\left(\int_{c}^{1-c}\left(J_{2}(\tau)\right)^{p}\left(\varepsilon_{0}^{p} u(\tau)-C_{4}\right) d \tau\right) d s-C_{3} \\
& \geq C_{1} \varepsilon_{0}^{p} \gamma_{1} \gamma_{2}^{p}\left(\int_{c}^{1-c} J_{1}(s) d s\right)\left(\int_{c}^{1-c}\left(J_{2}(\tau)\right)^{p} u(\tau) d \tau\right)-C_{5} \\
& \geq C_{1} \varepsilon_{0}^{p} \gamma_{1} \gamma_{2}^{p}\left(\int_{c}^{1-c} J_{1}(s) d s\right)\left(\int_{c}^{1-c}\left(J_{2}(\tau)\right)^{p} d \tau\right) \cdot \inf _{\tau \in[c, 1-c]} u(\tau)-C_{5} \\
& =2 \inf _{\tau \in[c, 1-c]} u(\tau)-C_{5},
\end{aligned}
$$

where $C_{5}=C_{3}+C_{1} C_{4} m_{1} m_{2} \gamma_{1} \gamma_{2}^{p}>0$.

Hence, $\inf _{t \in[c, 1-c]} u(t) \geq 2 \inf _{t \in[c, 1-c]} u(t)-C_{5}$, and so

$$
\inf _{t \in[c, 1-c]} u(t) \leq C_{5}, \quad \forall u \in M .
$$

Now from relations (10) and (12), one obtains $\|u\| \leq\left(\inf _{t \in[c, 1-c]} u(t)\right) / \gamma \leq C_{5} / \gamma$, for all $u \in M$, that is, $M$ is a bounded subset of $X$. 
Besides, there exists a sufficiently large $L>0$ such that

$$
u \neq \mathcal{A} u+\lambda u_{0}, \quad \forall u \in \partial B_{L} \cap P, \forall \lambda \geq 0 .
$$

From [2], we deduce that the fixed point index of the operator $\mathcal{A}$ over $B_{L} \cap P$ with respect to $P$ is

$$
i\left(\mathcal{A}, B_{L} \cap P, P\right)=0 .
$$

Next, from assumption (H4), we conclude that there exist $M_{0}>0$ and $\delta_{1} \in(0,1)$ such that

$$
\begin{array}{ll}
f(t, u) \leq M_{0} u^{q}, & \forall(t, u) \in[0,1] \times[0,1] ; \\
g(t, u) \leq \varepsilon_{1} u^{1 / q}, & \forall(t, u) \in[0,1] \times\left[0, \delta_{1}\right],
\end{array}
$$

where $\varepsilon_{1}=\min \left\{1 / M_{2},\left(1 /\left(2 M_{0} M_{1} M_{2}^{q}\right)\right)^{1 / q}\right\}>0, M_{1}=\int_{0}^{1} J_{1}(s) d s>0, M_{2}=\int_{0}^{1} J_{2}(s) d s>0$. Hence, for any $u \in \bar{B}_{\delta_{1}} \cap P$ and $t \in[0,1]$, we obtain

$$
\int_{0}^{1} G_{2}(t, s) g(s, u(s)) d s \leq \varepsilon_{1} \int_{0}^{1} J_{2}(s)(u(s))^{1 / q} d s \leq \varepsilon_{1} M_{2}\|u\|^{1 / q} \leq 1 .
$$

Therefore, by (14) and (15), we deduce that for any $u \in \bar{B}_{\delta_{1}} \cap P$ and $t \in[0,1]$

$$
\begin{aligned}
(\mathcal{A} u)(t) & \leq M_{0} \int_{0}^{1} G_{1}(t, s)\left(\int_{0}^{1} G_{2}(s, \tau) g(\tau, u(\tau)) d \tau\right)^{q} d s \\
& \leq M_{0} \varepsilon_{1}^{q} M_{2}^{q}\|u\| \int_{0}^{1} J_{1}(s) d s=M_{0} \varepsilon_{1}^{q} M_{1} M_{2}^{q}\|u\| \leq \frac{1}{2}\|u\| .
\end{aligned}
$$

This implies that $\|\mathcal{A} u\| \leq\|u\| / 2$ for all $u \in \partial B_{\delta_{1}} \cap P$. From [2], we conclude that the fixed point index of the operator $\mathcal{A}$ over $B_{\delta_{1}} \cap P$ with respect to $P$ is

$$
i\left(\mathcal{A}, B_{\delta_{1}} \cap P, P\right)=1 .
$$

Combining (13) and (16), we obtain

$$
i\left(\mathcal{A},\left(B_{L} \backslash \bar{B}_{\delta_{1}}\right) \cap P, P\right)=i\left(\mathcal{A}, B_{L} \cap P, P\right)-i\left(\mathcal{A}, B_{\delta_{1}} \cap P, P\right)=-1 .
$$

We deduce that $\mathcal{A}$ has at least one fixed point $u_{1} \in\left(B_{L} \backslash \bar{B}_{\delta_{1}}\right) \cap P$, that is, $\delta_{1}<\left\|u_{1}\right\|<L$.

Let $v_{1}(t)=\int_{0}^{1} G_{2}(t, s) g\left(s, u_{1}(s)\right) d s$. Then $\left(u_{1}, v_{1}\right) \in P \times P$ is a solution of (S)-(BC). In addition $\left\|v_{1}\right\|>0$. Indeed, if we suppose that $v_{1}(t)=0$, for all $t \in[0,1]$, then by using (H2) we have $f\left(s, v_{1}(s)\right)=f(s, 0)=0$, for all $s \in[0,1]$. This implies $u_{1}(t)=\int_{0}^{1} G_{1}(t, s) f\left(s, v_{1}(s)\right) d s=0$, for all $t \in[0,1]$, which contradicts $\left\|u_{1}\right\|>0$. The proof of Theorem 3.1 is completed.

Using similar arguments as those used in the proofs of Theorem 3.2 and Theorem 3.3 in [4], we also obtain the following results for our problem (S)-(BC).

Theorem 3.2 Assume that (H1)-(H2) hold. If the functions $f$ and $g$ also satisfy the conditions: 
(H5) There exists a positive constant $r \in(0, \infty)$ such that

(i) $f_{\infty}^{s}=\limsup _{u \rightarrow \infty} \sup _{t \in[0,1]} \frac{f(t, u)}{u^{r}} \in[0, \infty)$;

(ii) $g_{\infty}^{s}=\limsup _{u \rightarrow \infty} \sup _{t \in[0,1]} \frac{g(t, u)}{u^{1 / r}}=0$.

(H6) There exists $c \in(0,1 / 2)$ such that

(i) $f_{0}^{i}=\liminf _{u \rightarrow 0^{+}} \inf _{t \in[c, 1-c]} \frac{f(t, u)}{u} \in(0, \infty]$;

(ii) $g_{0}^{i}=\liminf _{u \rightarrow 0^{+}} \inf _{t \in[c, 1-c]} \frac{g(t, u)}{u}=\infty$,

then the problem $(\mathrm{S})-(\mathrm{BC})$ has at least one positive solution $(u(t), v(t)), t \in[0,1]$.

Theorem 3.3 Assume that (H1)-(H3), and (H6) hold. If the functions $f$ and $g$ also satisfy the condition:

(H7) For each $t \in[0,1], f(t, u)$ and $g(t, u)$ are nondecreasing with respect to $u$, and there exists a constant $N>0$ such that

$$
f\left(t, m_{0} \int_{0}^{1} g(s, N) d s\right)<\frac{N}{m_{0}}, \quad \forall t \in[0,1]
$$

where $m_{0}=\max \left\{K_{1}, K_{2}\right\}, K_{1}=\max _{s \in[0,1]} J_{1}(s), K_{2}=\max _{s \in[0,1]} J_{2}(s)$, and $J_{1}, J_{2}$ are defined in Section 2, then the problem (S)-(BC) has at least two positive solutions $\left(u_{1}(t), v_{1}(t)\right),\left(u_{2}(t), v_{2}(t)\right), t \in[0,1]$

\section{The singular case}

In this section, we investigate the existence of positive solutions for our problem (S)-(BC) under various assumptions on functions $f$ and $g$ which may be singular at $t=0$ and/or $t=1$.

The basic assumptions used here are the following.

$(\widetilde{\mathrm{H} 1}) \equiv(\mathrm{H} 1)$.

( $\widetilde{\mathrm{H} 2})$ The functions $f, g \in C\left((0,1) \times \mathbb{R}_{+}, \mathbb{R}_{+}\right)$and there exist $p_{i} \in C\left((0,1), \mathbb{R}_{+}\right), q_{i} \in C\left(\mathbb{R}_{+}\right.$, $\left.\mathbb{R}_{+}\right), i=1,2$, with $0<\int_{0}^{1} p_{i}(t) d t<\infty, i=1,2, q_{1}(0)=0, q_{2}(0)=0$ such that

$$
f(t, x) \leq p_{1}(t) q_{1}(x), \quad g(t, x) \leq p_{2}(t) q_{2}(x), \quad \forall t \in(0,1), x \in \mathbb{R}_{+} .
$$

We consider the Banach space $X=C([0,1])$ with supremum norm and define the cone $P \subset X$ by $P=\{u \in X, u(t) \geq 0, \forall t \in[0,1]\}$. We also define the operator $\widetilde{\mathcal{A}}: P \rightarrow X$ by

$$
(\widetilde{\mathcal{A}} u)(t)=\int_{0}^{1} G_{1}(t, s) f\left(s, \int_{0}^{1} G_{2}(s, \tau) g(\tau, u(\tau)) d \tau\right) d s
$$

Lemma 4.1 Assume that $(\widetilde{\mathrm{H} 1})-(\widetilde{\mathrm{H} 2})$ hold. Then $\widetilde{\mathcal{A}}: P \rightarrow P$ is completely continuous. 
Proof We denote by $\widetilde{\alpha}=\int_{0}^{1} J_{1}(s) p_{1}(s) d s$ and $\widetilde{\beta}=\int_{0}^{1} J_{2}(s) p_{2}(s) d s$. Using $(\widetilde{\mathrm{H} 2})$, we deduce that $0<\widetilde{\alpha}<\infty$ and $0<\widetilde{\beta}<\infty$. By Lemma 2.6 and the corresponding lemma for $G_{2}$, we see that $\widetilde{\mathcal{A}}$ maps $P$ into $P$.

We shall prove that $\widetilde{\mathcal{A}}$ maps bounded sets into relatively compact sets. Suppose $D \subset P$ is an arbitrary bounded set. Then there exists $\bar{M}_{1}>0$ such that $\|u\| \leq \bar{M}_{1}$ for all $u \in D$. By using $(\widetilde{\mathrm{H} 2})$ and Lemma 2.7, we obtain $\|\widetilde{\mathcal{A}} u\| \leq \widetilde{\alpha} \bar{M}_{3}$ for all $u \in D$, where $\bar{M}_{3}=$ $\sup _{x \in\left[0, \widetilde{\beta} \bar{M}_{2}\right]} q_{1}(x)$, and $\bar{M}_{2}=\sup _{x \in\left[0, \bar{M}_{1}\right]} q_{2}(x)$. In what follows, we shall prove that $\widetilde{\mathcal{A}}(D)$ is equicontinuous. By using Lemma 2.4, we have

$$
\begin{aligned}
(\widetilde{\mathcal{A}} u)(t)= & \int_{0}^{1} G_{1}(t, s) f\left(s, \int_{0}^{1} G_{2}(s, \tau) g(\tau, u(\tau)) d \tau\right) d s \\
= & \int_{0}^{1}\left[g_{1}(t, s)+\frac{t^{\alpha-1}}{\Delta_{1}} \int_{0}^{1} g_{1}(\tau, s) d H(\tau)\right] f\left(s, \int_{0}^{1} G_{2}(s, \tau) g(\tau, u(\tau)) d \tau\right) d s \\
= & \frac{1}{\Gamma(\alpha)} \int_{0}^{t}\left[t^{\alpha-1}(1-s)^{\alpha-1}-(t-s)^{\alpha-1}\right] f\left(s, \int_{0}^{1} G_{2}(s, \tau) g(\tau, u(\tau)) d \tau\right) d s \\
& +\frac{1}{\Gamma(\alpha)} \int_{t}^{1} t^{\alpha-1}(1-s)^{\alpha-1} f\left(s, \int_{0}^{1} G_{2}(s, \tau) g(\tau, u(\tau)) d \tau\right) d s \\
& +\frac{t^{\alpha-1}}{\Delta_{1}} \int_{0}^{1}\left(\int_{0}^{1} g_{1}(\tau, s) d H(\tau)\right) \\
& \times f\left(s, \int_{0}^{1} G_{2}(s, \tau) g(\tau, u(\tau)) d \tau\right) d s, \quad \forall t \in[0,1] .
\end{aligned}
$$

Therefore, for any $t \in(0,1)$, we obtain

$$
\begin{aligned}
(\widetilde{\mathcal{A}} u)^{\prime}(t)= & \frac{1}{\Gamma(\alpha)} \int_{0}^{t}\left[(\alpha-1) t^{\alpha-2}(1-s)^{\alpha-1}-(\alpha-1)(t-s)^{\alpha-2}\right] \\
& \times f\left(s, \int_{0}^{1} G_{2}(s, \tau) g(\tau, u(\tau)) d \tau\right) d s \\
& +\frac{1}{\Gamma(\alpha)} \int_{t}^{1}(\alpha-1) t^{\alpha-2}(1-s)^{\alpha-1} f\left(s, \int_{0}^{1} G_{2}(s, \tau) g(\tau, u(\tau)) d \tau\right) d s \\
& +\frac{(\alpha-1) t^{\alpha-2}}{\Delta_{1}} \int_{0}^{1}\left(\int_{0}^{1} g_{1}(\tau, s) d H(\tau)\right) f\left(s, \int_{0}^{1} G_{2}(s, \tau) g(\tau, u(\tau)) d \tau\right) d s .
\end{aligned}
$$

So, for any $t \in(0,1)$, we deduce

$$
\begin{aligned}
\left|(\widetilde{\mathcal{A}} u)^{\prime}(t)\right| \leq & \frac{1}{\Gamma(\alpha-1)} \int_{0}^{t}\left[t^{\alpha-2}(1-s)^{\alpha-1}+(t-s)^{\alpha-2}\right] p_{1}(s) \\
& \times q_{1}\left(\int_{0}^{1} G_{2}(s, \tau) g(\tau, u(\tau)) d \tau\right) d s \\
& +\frac{1}{\Gamma(\alpha-1)} \int_{t}^{1} t^{\alpha-2}(1-s)^{\alpha-1} p_{1}(s) q_{1}\left(\int_{0}^{1} G_{2}(s, \tau) g(\tau, u(\tau)) d \tau\right) d s \\
& +\frac{(\alpha-1) t^{\alpha-2}}{\Delta_{1}} \int_{0}^{1}\left(\int_{0}^{1} g_{1}(\tau, s) d H(\tau)\right) p_{1}(s) \\
& \times q_{1}\left(\int_{0}^{1} G_{2}(s, \tau) g(\tau, u(\tau)) d \tau\right) d s
\end{aligned}
$$




$$
\begin{aligned}
\leq & \bar{M}_{3}\left(\frac{1}{\Gamma(\alpha-1)} \int_{0}^{t}\left[t^{\alpha-2}(1-s)^{\alpha-1}+(t-s)^{\alpha-2}\right] p_{1}(s) d s\right. \\
& +\frac{1}{\Gamma(\alpha-1)} \int_{t}^{1} t^{\alpha-2}(1-s)^{\alpha-1} p_{1}(s) d s \\
& \left.+\frac{(\alpha-1) t^{\alpha-2}}{\Delta_{1}} \int_{0}^{1}\left(\int_{0}^{1} g_{1}(\tau, s) d H(\tau)\right) p_{1}(s) d s\right) .
\end{aligned}
$$

We denote

$$
\begin{aligned}
h(t)= & \frac{1}{\Gamma(\alpha-1)} \int_{0}^{t}\left[t^{\alpha-2}(1-s)^{\alpha-1}+(t-s)^{\alpha-2}\right] p_{1}(s) d s \\
& +\frac{1}{\Gamma(\alpha-1)} \int_{t}^{1} t^{\alpha-2}(1-s)^{\alpha-1} p_{1}(s) d s \\
\mu(t)= & h(t)+\frac{(\alpha-1) t^{\alpha-2}}{\Delta_{1}} \int_{0}^{1}\left(\int_{0}^{1} g_{1}(\tau, s) d H(\tau)\right) p_{1}(s) d s, \quad t \in(0,1) .
\end{aligned}
$$

For the integral of the function $h$, by exchanging the order of integration, we obtain

$$
\begin{aligned}
\int_{0}^{1} h(t) d t= & \frac{1}{\Gamma(\alpha-1)} \int_{0}^{1}\left(\int_{0}^{t}\left[t^{\alpha-2}(1-s)^{\alpha-1}+(t-s)^{\alpha-2}\right] p_{1}(s) d s\right) d t \\
& +\frac{1}{\Gamma(\alpha-1)} \int_{0}^{1}\left(\int_{t}^{1} t^{\alpha-2}(1-s)^{\alpha-1} p_{1}(s) d s\right) d t \\
= & \frac{1}{\Gamma(\alpha-1)} \int_{0}^{1}\left(\int_{s}^{1}\left[t^{\alpha-2}(1-s)^{\alpha-1}+(t-s)^{\alpha-2}\right] p_{1}(s) d t\right) d s \\
& +\frac{1}{\Gamma(\alpha-1)} \int_{0}^{1}\left(\int_{0}^{s} t^{\alpha-2}(1-s)^{\alpha-1} p_{1}(s) d t\right) d s \\
= & \frac{2}{\Gamma(\alpha)} \int_{0}^{1}(1-s)^{\alpha-1} p_{1}(s) d s<\infty
\end{aligned}
$$

For the integral of the function $\mu$, we have

$$
\begin{aligned}
\int_{0}^{1} \mu(t) d t & =\int_{0}^{1} h(t) d t+\frac{\alpha-1}{\Delta_{1}}\left(\int_{0}^{1}\left(\int_{0}^{1} g_{1}(\tau, s) d H(\tau)\right) p_{1}(s) d s\right)\left(\int_{0}^{1} t^{\alpha-2} d t\right) \\
& \leq \frac{2}{\Gamma(\alpha)} \int_{0}^{1}(1-s)^{\alpha-1} p_{1}(s)+\frac{H(1)-H(0)}{\Delta_{1}} \int_{0}^{1} g_{1}\left(\theta_{1}(s), s\right) p_{1}(s) d s \\
& \leq \frac{1}{\Gamma(\alpha)}\left(2+\frac{H(1)-H(0)}{\Delta_{1}}\right) \int_{0}^{1}(1-s)^{\alpha-1} p_{1}(s) d s<\infty .
\end{aligned}
$$

We deduce that $\mu \in L^{1}(0,1)$. Thus for any given $t_{1}, t_{2} \in[0,1]$ with $t_{1} \leq t_{2}$ and $u \in D$, by (17), we conclude

$$
\left|(\widetilde{\mathcal{A}} u)\left(t_{1}\right)-(\widetilde{\mathcal{A}} u)\left(t_{2}\right)\right|=\left|\int_{t_{1}}^{t_{2}}(\widetilde{\mathcal{A}} u)^{\prime}(t) d t\right| \leq \bar{M}_{3} \int_{t_{1}}^{t_{2}} \mu(t) d t .
$$

From (18), (19), and the absolute continuity of the integral function, we find that $\widetilde{\mathcal{A}}(D)$ is equicontinuous. By the Ascoli-Arzelà theorem, we deduce that $\widetilde{\mathcal{A}}(D)$ is relatively compact. 
Therefore $\widetilde{\mathcal{A}}$ is a compact operator. Besides, we can easily show that $\widetilde{\mathcal{A}}$ is continuous on $P$. Hence $\widetilde{\mathcal{A}}: P \rightarrow P$ is completely continuous.

Theorem 4.1 Assume that $(\widetilde{\mathrm{H} 1})-(\widetilde{\mathrm{H} 2})$ hold. If the functions $f$ and $g$ also satisfy the conditions:

( $\widetilde{\mathrm{H}} 3)$ There exist $\alpha_{1}, \alpha_{2} \in(0, \infty)$ with $\alpha_{1} \alpha_{2} \leq 1$ such that

$$
\text { (i) } q_{1 \infty}^{s}=\limsup _{x \rightarrow \infty} \frac{q_{1}(x)}{x^{\alpha_{1}}} \in[0, \infty) ; \quad \text { (ii) } \quad q_{2 \infty}^{s}=\limsup _{x \rightarrow \infty} \frac{q_{2}(x)}{x^{\alpha_{2}}}=0 .
$$

( $\widetilde{\mathrm{H}} 4)$ There exist $\beta_{1}, \beta_{2} \in(0, \infty)$ with $\beta_{1} \beta_{2} \leq 1$ and $c \in\left(0, \frac{1}{2}\right)$ such that

$$
\text { (i) } \tilde{f}_{0}^{i}=\liminf _{x \rightarrow 0^{+}} \inf _{t \in[c, 1-c]} \frac{f(t, x)}{x^{\beta_{1}}} \in(0, \infty] ; \quad \text { (ii) } \tilde{g}_{0}^{i}=\liminf _{x \rightarrow 0^{+}} \inf _{t \in[c, 1-c]} \frac{g(t, x)}{x^{\beta_{2}}}=\infty \text {, }
$$

then the problem (S)-(BC) has at least one positive solution $(u(t), v(t)), t \in[0,1]$.

Proof Because the proof of this theorem is similar to that of Theorem 3 in [5], we will sketch some parts of it. For $c$ given in $(\widetilde{\mathrm{H} 4})$, we consider the cone $P_{0}=\{u \in X, u(t) \geq$ $\left.0, \forall t \in[0,1], \min _{t \in[c, 1-c]} u(t) \geq \gamma\|u\|\right\}$, where $\gamma=\min \left\{\gamma_{1}, \gamma_{2}\right\}$. Under assumptions ( $\widetilde{\mathrm{H} 1}$ )$(\widetilde{\mathrm{H} 2})$, we obtain $\widetilde{\mathcal{A}}(P) \subset P_{0}$. By $(\widetilde{\mathrm{H} 3})$, we deduce that there exist $C_{6}, C_{7}, C_{8}>0$ and $\varepsilon_{2} \in$ $\left(0,\left(2^{\alpha_{1}} C_{6} \widetilde{\alpha} \widetilde{\beta}^{\alpha_{1}}\right)^{-1 / \alpha_{1}}\right)$ such that

$$
q_{1}(x) \leq C_{6} x^{\alpha_{1}}+C_{7}, \quad q_{2}(x) \leq \varepsilon_{2} x^{\alpha_{2}}+C_{8}, \quad \forall x \in[0, \infty) .
$$

By using $(20)$ and $(\widetilde{\mathrm{H} 2})$, for any $u \in P_{0}$, we conclude

$$
\begin{aligned}
(\widetilde{\mathcal{A}} u)(t) \leq & \int_{0}^{1} G_{1}(t, s) p_{1}(s) q_{1}\left(\int_{0}^{1} G_{2}(s, \tau) g(\tau, u(\tau)) d \tau\right) d s \\
\leq & C_{6} \int_{0}^{1} G_{1}(t, s) p_{1}(s)\left(\int_{0}^{1} G_{2}(s, \tau) g(\tau, u(\tau)) d \tau\right)^{\alpha_{1}} d s \\
& +C_{7} \int_{0}^{1} J_{1}(s) p_{1}(s) d s \\
\leq & C_{6} \int_{0}^{1} J_{1}(s) p_{1}(s)\left[\int_{0}^{1} G_{2}(s, \tau) p_{2}(\tau)\left(\varepsilon_{2}(u(\tau))^{\alpha_{2}}+C_{8}\right) d \tau\right]^{\alpha_{1}} d s+\widetilde{\alpha} C_{7} \\
\leq & C_{6}\left(\int_{0}^{1} J_{1}(s) p_{1}(s) d s\right)\left(\int_{0}^{1} J_{2}(\tau) p_{2}(\tau) d \tau\right)^{\alpha_{1}}\left(\varepsilon_{2}\|u\|^{\alpha_{2}}+C_{8}\right)^{\alpha_{1}}+\widetilde{\alpha} C_{7} \\
\leq & C_{6} 2^{\alpha_{1}} \varepsilon_{2}^{\alpha_{1}} \widetilde{\alpha} \widetilde{\beta}^{\alpha_{1}}\|u\|^{\alpha_{1} \alpha_{2}}+C_{6} 2^{\alpha_{1}} \widetilde{\alpha} \widetilde{\beta}^{\alpha_{1}} C_{8}^{\alpha_{1}}+\widetilde{\alpha} C_{7}, \quad \forall t \in[0,1] .
\end{aligned}
$$

By the definition of $\varepsilon_{2}$, we can choose sufficiently large $R_{1}>0$ such that

$$
\|\widetilde{\mathcal{A}} u\| \leq\|u\|, \quad \forall u \in \partial B_{R_{1}} \cap P_{0} .
$$

From $(\widetilde{\mathrm{H}} 4)$, we deduce that there exist positive constants $C_{9}>0, x_{1}>0$, and $\varepsilon_{3} \geq$ $\left(1 /\left(C_{9} \gamma_{1} \gamma_{2}^{\beta_{1}} \gamma^{\beta_{1} \beta_{2}} \bar{\theta}_{1} \bar{\theta}_{2}^{\beta_{1}}\right)\right)^{1 / \beta_{1}}$ such that

$$
f(t, x) \geq C_{9} x^{\beta_{1}}, \quad g(t, x) \geq \varepsilon_{3} x^{\beta_{2}}, \quad \forall(t, x) \in[c, 1-c] \times\left[0, x_{1}\right],
$$


where $\bar{\theta}_{1}=\int_{c}^{1-c} J_{1}(s) d s$ and $\bar{\theta}_{2}=\int_{c}^{1-c} J_{2}(s) d s$. From the assumption $q_{2}(0)=0$ and the continuity of $q_{2}$, we conclude that there exists sufficiently small $\varepsilon_{4} \in\left(0, \min \left\{x_{1}, 1\right\}\right)$ such that $q_{2}(x) \leq \widetilde{\beta}^{-1} x_{1}$ for all $x \in\left[0, \varepsilon_{4}\right]$, where $\widetilde{\beta}=\int_{0}^{1} J_{2}(s) p_{2}(s) d s$. Therefore for any $u \in \partial B_{\varepsilon_{4}} \cap P_{0}$ and $s \in[0,1]$, we have

$$
\int_{0}^{1} G_{2}(s, \tau) g(\tau, u(\tau)) d \tau \leq \int_{0}^{1} J_{2}(\tau) p_{2}(\tau) q_{2}(u(\tau)) d \tau \leq x_{1} .
$$

By (22), (23), Lemma 2.7, and Lemma 2.8, for any $u \in \partial B_{\varepsilon_{4}} \cap P_{0}$ and $t \in[c, 1-c]$, we obtain

$$
\begin{aligned}
(\widetilde{\mathcal{A}} u)(t) & \geq C_{9} \int_{c}^{1-c} G_{1}(t, s)\left(\int_{c}^{1-c} G_{2}(s, \tau) g(\tau, u(\tau)) d \tau\right)^{\beta_{1}} d s \\
& \geq C_{9} \int_{c}^{1-c} G_{1}(t, s)\left(\varepsilon_{3} \int_{c}^{1-c} G_{2}(s, \tau)(u(\tau))^{\beta_{2}} d \tau\right)^{\beta_{1}} d s \\
& \geq C_{9} \gamma_{1} \int_{c}^{1-c} J_{1}(s)\left(\left(\varepsilon_{3} \gamma_{2}\right)^{\beta_{1}}\left(\int_{c}^{1-c} J_{2}(\tau)(u(\tau))^{\beta_{2}} d \tau\right)^{\beta_{1}}\right) d s \\
& \geq C_{9} \gamma_{1} \gamma_{2}^{\beta_{1}} \varepsilon_{3}^{\beta_{1}} \gamma^{\beta_{1} \beta_{2}} \bar{\theta}_{1} \bar{\theta}_{2}^{\beta_{1}}\|u\|^{\beta_{1} \beta_{2}} \geq\|u\|^{\beta_{1} \beta_{2}} \geq\|u\| .
\end{aligned}
$$

Therefore

$$
\|\widetilde{\mathcal{A}} u\| \geq\|u\|, \quad \forall u \in \partial B_{\varepsilon_{4}} \cap P_{0} .
$$

By (21), (24), and the Guo-Krasnosel'skii fixed point theorem, we deduce that $\widetilde{\mathcal{A}}$ has at least one fixed point $u_{2} \in\left(\bar{B}_{R_{1}} \backslash B_{\varepsilon_{4}}\right) \cap P_{0}$. Then our problem (S)-(BC) has at least one positive solution $\left(u_{2}, v_{2}\right) \in P_{0} \times P_{0}$ where $v_{2}(t)=\int_{0}^{1} G_{2}(t, s) g\left(s, u_{2}(s)\right) d s$. The proof of Theorem 4.1 is completed.

Using similar arguments as those used in the proof of Theorem 2 in [5] (see also [14] for a particular case of the problem studied in [5]), we also obtain the following result for our problem $(\mathrm{S})-(\mathrm{BC})$.

Theorem 4.2 Assume that $(\widetilde{\mathrm{H} 1})-(\widetilde{\mathrm{H} 2})$ hold. If the functions $f$ and $g$ also satisfy the conditions:

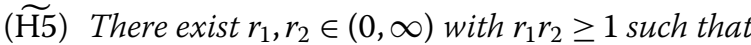
(i) $q_{10}^{s}=\limsup _{x \rightarrow 0^{+}} \frac{q_{1}(x)}{x^{r_{1}}} \in[0, \infty)$;
(ii) $q_{20}^{s}=\limsup _{x \rightarrow 0^{+}} \frac{q_{2}(x)}{x^{r_{2}}}=0$.

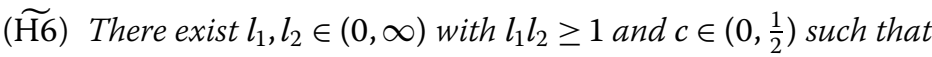

$$
\begin{aligned}
& \text { (i) } \widetilde{f}_{\infty}^{i}=\liminf _{x \rightarrow \infty} \inf _{t \in[c, 1-c]} \frac{f(t, x)}{x^{l_{1}}} \in(0, \infty] ; \\
& \text { (ii) } \tilde{g}_{\infty}^{i}=\liminf _{x \rightarrow \infty} \inf _{t \in[c, 1-c]} \frac{g(t, x)}{x^{l_{2}}}=\infty,
\end{aligned}
$$

then the problem (S)-(BC) has at least one positive solution $(u(t), v(t)), t \in[0,1]$. 


\section{Examples}

Let $\alpha=5 / 2(n=3), \beta=4 / 3(m=2)$,

$$
H(t)= \begin{cases}0, & t \in[0,1 / 4), \\ 2, & t \in[1 / 4,3 / 4), \\ 3, & t \in[3 / 4,1]\end{cases}
$$

and $K(t)=t^{3}$ for all $t \in[0,1]$. Then $\int_{0}^{1} u(s) d H(s)=2 u\left(\frac{1}{4}\right)+u\left(\frac{3}{4}\right)$ and $\int_{0}^{1} v(s) d K(s)=$ $3 \int_{0}^{1} s^{2} v(s) d s$.

We consider the system of fractional differential equations

$$
\left(\mathrm{S}_{0}\right) \quad \begin{cases}D_{0+}^{5 / 2} u(t)+f(t, v(t))=0, & t \in(0,1), \\ D_{0+}^{4 / 3} v(t)+g(t, u(t))=0, & t \in(0,1),\end{cases}
$$

with the boundary conditions

$$
\left(\mathrm{BC}_{0}\right) \quad\left\{\begin{array}{l}
u(0)=u^{\prime}(0)=0, \quad u(1)=2 u\left(\frac{1}{4}\right)+u\left(\frac{3}{4}\right), \\
v(0)=0, \quad v(1)=3 \int_{0}^{1} s^{2} v(s) d s
\end{array}\right.
$$

Then we obtain $\Delta_{1}=1-\int_{0}^{1} s^{\alpha-1} d H(s)=1-2\left(\frac{1}{4}\right)^{3 / 2}-\left(\frac{3}{4}\right)^{3 / 2}=\frac{3(2-\sqrt{3})}{8} \approx 0.1005>0, \Delta_{2}=$ $1-\int_{0}^{1} s^{\beta-1} d K(s)=1-3 \int_{0}^{1} s^{7 / 3} d s=\frac{1}{10}=0.1>0$. We also deduce

$$
\begin{aligned}
& g_{1}(t, s)=\frac{4}{3 \sqrt{\pi}} \begin{cases}t^{3 / 2}(1-s)^{3 / 2}-(t-s)^{3 / 2}, & 0 \leq s \leq t \leq 1, \\
t^{3 / 2}(1-s)^{3 / 2}, & 0 \leq t \leq s \leq 1,\end{cases} \\
& g_{2}(t, s)=\frac{1}{\Gamma(4 / 3)} \begin{cases}t^{1 / 3}(1-s)^{1 / 3}-(t-s)^{1 / 3}, & 0 \leq s \leq t \leq 1, \\
t^{1 / 3}(1-s)^{1 / 3}, & 0 \leq t \leq s \leq 1,\end{cases}
\end{aligned}
$$

$\theta_{1}(s)=\frac{1}{3-3 s+s^{2}}$ and $\theta_{2}(s)=s$ for all $s \in[0,1]$.

For the functions $J_{1}$ and $J_{2}$, we obtain

$$
\begin{aligned}
& J_{1}(s)=g_{1}\left(\theta_{1}(s), s\right)+\frac{1}{\Delta_{1}} \int_{0}^{1} g_{1}(\tau, s) d H(\tau) \\
& =g_{1}\left(\frac{1}{3-3 s+s^{2}}, s\right)+\frac{1}{\Delta_{1}}\left[2 g_{1}\left(\frac{1}{4}, s\right)+g_{1}\left(\frac{3}{4}, s\right)\right] \\
& =\left\{\begin{array}{rlrl}
\frac{4}{3 \sqrt{\pi}}\left\{\frac{s(1-s)^{3 / 2}}{\left(3-3 s+s^{2}\right)^{1 / 2}}+\frac{1}{3(2-\sqrt{3})}\left[2\left((1-s)^{3 / 2}-(1-4 s)^{3 / 2}\right)\right.\right. & \\
& \left.\left.+\left(3 \sqrt{3}(1-s)^{3 / 2}-(3-4 s)^{3 / 2}\right)\right]\right\}, & & 0 \leq s<\frac{1}{4}, \\
\frac{4}{3 \sqrt{\pi}}\left\{\frac{s(1-s)^{3 / 2}}{\left(3-3 s+s^{2}\right)^{1 / 2}}+\frac{1}{3(2-\sqrt{3})}\left[2(1-s)^{3 / 2}\right.\right. & & \\
& \left.\left.+\left(3 \sqrt{3}(1-s)^{3 / 2}-(3-4 s)^{3 / 2}\right)\right]\right\}, & & \frac{1}{4} \leq s<\frac{3}{4}, \\
\frac{4}{3 \sqrt{\pi}}\left[\frac{s(1-s)^{3 / 2}}{\left(3-3 s+s^{2}\right)^{1 / 2}}+\frac{(2+3 \sqrt{3})(1-s)^{3 / 2}}{3(2-\sqrt{3})}\right], & \frac{3}{4} \leq s \leq 1
\end{array}\right.
\end{aligned}
$$

and

$$
\begin{aligned}
J_{2}(s) & =g_{2}\left(\theta_{2}(s), s\right)+\frac{1}{\Delta_{2}} \int_{0}^{1} g_{2}(\tau, s) d K(\tau) \\
& =g_{2}(s, s)+\frac{3}{\Delta_{2}} \int_{0}^{1} \tau^{2} g_{2}(\tau, s) d \tau
\end{aligned}
$$




$$
\begin{aligned}
= & \frac{1}{\Gamma(4 / 3)}\left(s^{1 / 3}(1-s)^{1 / 3}+9(1-s)^{1 / 3}-9(1-s)^{10 / 3}-\frac{180}{7} s(1-s)^{7 / 3}\right. \\
& \left.-\frac{45}{2} s^{2}(1-s)^{4 / 3}\right), \quad s \in[0,1] .
\end{aligned}
$$

Example 1 We consider the functions

$$
f(t, u)=a\left(u^{\alpha_{0}}+u^{\beta_{0}}\right), \quad g(t, u)=b\left(u^{\gamma_{0}}+u^{\delta_{0}}\right), \quad t \in[0,1], u \geq 0,
$$

where $\alpha_{0}>1,0<\beta_{0}<1, \gamma_{0}>2,0<\delta_{0}<1, a, b>0$. We have $K_{1}=\max _{s \in[0,1]} J_{1}(s) \approx$ $1.8076209, K_{2}=\max _{s \in[0,1]} J_{2}(s) \approx 4.16640785$. Then $m_{0}=\max \left\{K_{1}, K_{2}\right\}=K_{2}$. The functions $f(t, u)$ and $g(t, u)$ are nondecreasing with respect to $u$, for any $t \in[0,1]$, and for $p=1 / 2$ and $c \in(0,1 / 2)$ the assumptions (H3) and (H6) are satisfied; indeed we obtain

$$
\begin{array}{ll}
f_{\infty}^{i}=\lim _{u \rightarrow \infty} \frac{a\left(u^{\alpha_{0}}+u^{\beta_{0}}\right)}{u^{1 / 2}}=\infty, & g_{\infty}^{i}=\lim _{u \rightarrow \infty} \frac{b\left(u^{\gamma_{0}}+u^{\delta_{0}}\right)}{u^{2}}=\infty, \\
f_{0}^{i}=\lim _{u \rightarrow 0^{+}} \frac{a\left(u^{\alpha_{0}}+u^{\beta_{0}}\right)}{u}=\infty, \quad g_{0}^{i}=\lim _{u \rightarrow 0^{+}} \frac{b\left(u^{\gamma_{0}}+u^{\delta_{0}}\right)}{u}=\infty .
\end{array}
$$

We take $N=1$ and then $\int_{0}^{1} g(s, 1) d s=2 b$ and $f\left(t, 2 b m_{0}\right)=a\left[\left(2 b m_{0}\right)^{\alpha_{0}}+\left(2 b m_{0}\right)^{\beta_{0}}\right]$. If $a\left[\left(2 b m_{0}\right)^{\alpha_{0}}+\left(2 b m_{0}\right)^{\beta_{0}}\right]<\frac{1}{m_{0}} \Leftrightarrow a\left[m_{0}^{\alpha_{0}+1}(2 b)^{\alpha_{0}}+m_{0}^{\beta_{0}+1}(2 b)^{\beta_{0}}\right]<1$, then the assumption (H7) is satisfied. For example, if $\alpha_{0}=3 / 2, \beta_{0}=1 / 3, b=1 / 2$, and $a<\frac{1}{m_{0}^{5 / 2}+m_{0}^{4 / 3}}$ (e.g. $a \leq$ $0.0237)$, then the above inequality is satisfied. By Theorem 3.3, we deduce that the problem $\left(\mathrm{S}_{0}\right)-\left(\mathrm{BC}_{0}\right)$ has at least two positive solutions.

Example 2 We consider the functions

$$
f(t, x)=\frac{x^{a}}{t^{\zeta_{1}}(1-t)^{\rho_{1}}}, \quad g(t, x)=\frac{x^{b}}{t^{\zeta_{2}}(1-t)^{\rho_{2}}},
$$

with $a, b>1$ and $\zeta_{1}, \rho_{1}, \zeta_{2}, \rho_{2} \in(0,1)$. Here $f(t, x)=p_{1}(t) q_{1}(x)$ and $g(t, x)=p_{2}(t) q_{2}(x)$, where

$$
p_{1}(t)=\frac{1}{t^{\zeta_{1}}(1-t)^{\rho_{1}}}, \quad p_{2}(t)=\frac{1}{t^{\zeta_{2}}(1-t)^{\rho_{2}}}, \quad q_{1}(x)=x^{a}, \quad q_{2}(x)=x^{b} .
$$

We have $0<\int_{0}^{1} p_{1}(s) d s<\infty, 0<\int_{0}^{1} p_{2}(s) d s<\infty$.

In ( $\widetilde{\mathrm{H}} 5)$, for $r_{1}<a, r_{2}<b$ and $r_{1} r_{2} \geq 1$, we obtain

$$
\limsup _{x \rightarrow 0^{+}} \frac{q_{1}(x)}{x^{r_{1}}}=0, \quad \limsup _{x \rightarrow 0^{+}} \frac{q_{2}(x)}{x^{r_{2}}}=0 .
$$

In ( $\widetilde{\mathrm{H}}$ ), for $l_{1}<a, l_{2}<b, l_{1} l_{2} \geq 1$, and $c \in\left(0, \frac{1}{2}\right)$, we have

$$
\liminf _{x \rightarrow \infty} \inf _{t \in[c, 1-c]} \frac{f(t, x)}{x^{l_{1}}}=\infty, \quad \liminf _{x \rightarrow \infty} \inf _{t \in[c, 1-c]} \frac{g(t, x)}{x^{l_{2}}}=\infty .
$$

For example, if $a=3 / 2, b=2, r_{1}=1, r_{2}=3 / 2, l_{1}=1, l_{2}=3 / 2$, the above conditions are satisfied. Then, by Theorem 4.2 , we deduce that the problem $\left(\mathrm{S}_{0}\right)-\left(\mathrm{BC}_{0}\right)$ has at least one positive solution. 


\section{Competing interests}

The authors declare that they have no competing interests.

\section{Authors' contributions}

Both authors contributed equally to this paper. Both authors read and approved the final manuscript.

\section{Author details}

${ }^{1}$ Department of Mathematics, Baylor University, Waco, TX 76798-7328, USA. ${ }^{2}$ Department of Mathematics, Gh. Asachi Technical University, lasi, 700506, Romania.

\section{Acknowledgements}

The work of R Luca was supported by the CNCS grant PN-II-ID-PCE-2011-3-0557, Romania

Received: 8 January 2014 Accepted: 11 March 2014 Published: 20 Mar 2014

\section{References}

1. Guo, D, Lakshmikantham, V: Nonlinear Problems in Abstract Cones. Academic Press, New York (1988)

2. Amann, H: Fixed point equations and nonlinear eigenvalue problems in ordered Banach spaces. SIAM Rev. 18 620-709 (1976)

3. Zhou, Y, Xu, Y: Positive solutions of three-point boundary value problems for systems of nonlinear second order ordinary differential equations. J. Math. Anal. Appl. 320, 578-590 (2006)

4. Henderson, J, Luca, R: Existence and multiplicity for positive solutions of a system of higher-order multi-point boundary value problems. Nonlinear Differ. Equ. Appl. 20(3), 1035-1054 (2013)

5. Henderson, J, Luca, R: Positive solutions for singular systems of higher-order multi-point boundary value problems. Math. Model. Anal. 18(3), 309-324 (2013)

6. Henderson, J, Luca, R: Positive solutions for a system of nonlocal fractional boundary value problems. Fract. Calc. Appl. Anal. 16(4), 985-1008 (2013)

7. Baleanu, D, Diethelm, K, Scalas, E, Trujillo, JJ: Fractional Calculus Models and Numerical Methods. Series on Complexity, Nonlinearity and Chaos. World Scientific, Boston (2012)

8. Das, S: Functional Fractional Calculus for System Identification and Controls. Springer, New York (2008)

9. Graef, JR, Kong, L, Kong, Q, Wang, M: Uniqueness of positive solutions of fractional boundary value problems with non-homogeneous integral boundary conditions. Fract. Calc. Appl. Anal. 15(3), 509-528 (2012)

10. Kilbas, AA, Srivastava, HM, Trujillo, JJ: Theory and Applications of Fractional Differential Equations. North-Holland Mathematics Studies, vol. 204. Elsevier, Amsterdam (2006)

11. Podlubny, I: Fractional Differential Equations. Academic Press, San Diego (1999)

12. Sabatier, J, Agrawal, OP, Machado, JAT (eds.): Advances in Fractional Calculus: Theoretical Developments and Applications in Physics and Engineering. Springer, Dordrecht (2007)

13. Samko, SG, Kilbas, AA, Marichev, Ol: Fractional Integrals and Derivatives: Theory and Applications. Gordon \& Breach, Yverdon (1993)

14. Liu, B, Liu, L, Wu, Y: Positive solutions for singular systems of three-point boundary value problems. Comput. Math. Appl. 53, 1429-1438 (2007)

$10.1186 / 1687-2770-2014-60$

Cite this article as: Henderson and Luca: Existence and multiplicity of positive solutions for a system of fractional boundary value problems. Boundary Value Problems 2014, 2014:60

\section{Submit your manuscript to a SpringerOpen ${ }^{\ominus}$ journal and benefit from:}

- Convenient online submission

- Rigorous peer review

Immediate publication on acceptance

Open access: articles freely available online

- High visibility within the field

- Retaining the copyright to your article 